\title{
Uso sustentable de la Microcuenca del Río Blanco: Posibles soluciones desde un Programa de Manejo Ambiental.
}

Sustainable use of the Río Blanco Micro-basin: Possible solutions from an Environmental Management Program.

\author{
María Soledad Nuñez Moreno. ${ }^{1} \&$ Cristina Gabriela Calderón Tapia. ${ }^{2}$ \\ Recibido: 13-11-2020 / Revisado: 12-12-2020 /Aceptado: 14-01-2021/ Publicado: 05-02-2021
}

\begin{abstract}
.
DOI: https://doi.org/10.33262/concienciadigital.v4i1.1.1560

Introduction. The progressive deterioration that occurs worldwide as a consequence of the indiscriminate use of natural resources and the environment, constitutes a serious problem, which demands urgent responses directed to the management and sustainability of the environment. Ecuador, regardless of the efforts made, does not escape this reality. An example of this is the use of the Rio Blanco Micro-basin, of the Penipe canton, of Chimborazo. Objective. Provide an Environmental Management Program, which allows in parallel the protection of the water resources of the micro-basin and in turn contributes to sustainable local development. Methodology. The study followed a qualitative, descriptive, non-experimental methodology, accompanied by theoretical and empirical methods. The investigative procedure was framed in three stages: Verification and diagnosis of the problem under study, determination of the objectives and strategy to be followed and design of the Environmental Management Program. Results. 1. The existence of the problem under study and the need to search for alternatives that guarantee the sustainable use of the reference micro-basin are corroborated. 2. An Environmental Management Program is provided, which, given its structure, conformation and planned schedule for its implementation, allows the protection of the water resources of the "Río Blanco" micro-basin and in turn contributes to sustainable local development. Conclusions. The conservation and proper management of environmental resources, in addition to being an extremely high priority, requires a timely intervention process, which must be supported by a comprehensive Environmental Management Plan, which favors not only the protection of water resources. , but also the sustainability and local development of the Río Blanco micro-basin ", this will allow improving the conservation and availability of natural resources as well as the survival of the different species that make up the ecosystem, including human life.
\end{abstract}

\footnotetext{
${ }^{1}$ Escuela Superior Politécnica de Chimborazo, Facultad de Ciencias, Riobamba, Ecuador, solenu0408@gmail.com, ORCID: https://orcid.org/0000-0001-7526-9870

${ }^{2}$ Escuela Superior Politécnica de Chimborazo, Facultad de Ciencias, Riobamba, Ecuador, cristy.gct@gmail.com, ORCID: https://orcid.org/0000-0002-8574-103X
} 
Keywords: Río Blanco micro-basin, Environmental Management Program, sustainable use.

\section{Resumen}

Introducción. El deterioro progresivo que a nivel mundial se da como consecuencia del uso indiscriminado de los recursos naturales y del medio ambiente, constituye un grave problema, el cual demanda de urgentes respuestas direccionadas al manejo y sustentabilidad del medio ambiente. Ecuador, independientemente de los esfuerzos realizados no escapa de esta realidad. Un ejemplo de esto lo constituye el uso de la Microcuenca Rio Blanco, del cantón Penipe, de Chimborazo. Objetivo. Aportar un Programa de Manejo Ambiental, que permita de manera paralela la protección de los recursos hídricos de la microcuenca y a su vez tribute al desarrollo local sostenible. Metodología. El estudio siguió una metodología cualitativa, de tipo descriptiva, no experimental, acompañada de métodos teóricos y empíricos. El proceder investigativo se enmarco en tres etapas: Constatación y diagnóstico del problema objeto de estudio, determinación de los objetivos y estrategia a seguir y diseño del Programa de Manejo Ambiental. Resultados. 1. Se corrobora la existencia del problema objeto de estudio y la necesidad de buscar alternativas que garanticen el uso sustentable de la microcuenca de referencia. 2. Se aporta un Programa de Manejo Ambiental, que dada su estructura, conformación y cronograma previsto para su implementación permite la protección de los recursos hídricos de la microcuenca "Río Blanco" y a su vez tributa al desarrollo local sostenible. Conclusiones. La conservación y manejo adecuado de los recursos ambientales, además de ser en extremo prioritaria requiere de un proceso de intervención oportuno, el cual debe estar sustentado en un Plan de Manejo Ambiental, de carácter integral, que favorezca no sólo la protección de los recursos hídricos, sino también la sostenibilidad y desarrollo local de la microcuenca Río Blanco”, ello permitirá mejorar la conservación y disponibilidad de los recursos naturales así como la supervivencia de las diferentes especies que conforman el ecosistema, incluyendo la vida humana.

Palabras clave: microcuenca del Río Blanco, Programa de Manejo Ambiental, uso sustentable.

\section{Introducción}

Para nadie es un secreto el grave deterioro que se viene dando a nivel mundial como consecuencia del uso indiscriminado de los recursos naturales y del medio ambiente. Hoy la población enfrenta los lamentables efectos causados por el irrespeto a la naturaleza y a la madre tierra. Los cambios ocurridos por el cambio climático, los desastres naturales y la desproporción existente sobre el uso improcedente del medio ambiente, ha traído serias dificultades a la humanidad y lo que es peor, sus daños marcaran por siempre la supervivencia del hombre y de las diferentes especies en la tierra. 
Es por ello que resulta trascendental buscar alternativas que favorezcan el uso correcto de los recursos naturales, con especial interés en el cuidado y protección del medio ambiente. Dada esta situación lo anterior se ha convertido desde hace unos años acá, en una de las tareas más importantes y acciones a desarrollar por cada gobierno a nivel internacional y por supuesto nacional. Sin embargo, la realidad existe en las diferentes latitudes exponen situaciones muy complejas, llegando incluso a comprometer la vida en el planeta tierra, de lo cual Ecuador como país tampoco escapa.

A juicio de López, (2014), asegurar la calidad de vida y el desarrollo sostenible de nuestras sociedades exige en la actualidad una gestión racional y equilibrada de los recursos naturales. En esta misma línea Arteta, Moreno, \& Steffanell, (2015), explican que los problemas ambientales aparecen por el resultado de diferentes interacciones entre el hombre y el territorio; asumiendo que muchos son consecuencias o resultado de un uso inadecuado de estos recursos.

Esto nos hace compartir con los autores precedentes en una idea fundamental, resulta primordial educar a la población en estos nuevos escenarios, de modo tal que tengan un proceder de actuación responsable y coherente con la protección y cuidado de los recursos ambientales; se trata por tanto de lograr que desde las primeras edades aprendamos a tener una actuación responsable y ética en función de promover un comportamiento social responsable que favorezca la protección de los recursos naturales.

En correspondencia con lo señalado somos del criterio que una de las acciones a considerar está relacionada con el desarrollo sustentable de las cuencas hidrográficas, las cuales ocupan la atención de muchos investigadores, precisamente por sus bondades naturales y los aportes que ofrecen al desarrollo integral de todos.

Al respecto coincidimos con Sepúlveda, (2002) al destacar que las cuencas son proveedoras de servicios ambientales, los cuales además de ser cada vez más escasos dependen en gran medida de la importancia que se les conceda los propios beneficiarios.

En este ámbito, son muy importantes los aportes de Van denBrink et al., (2007) y Baran y Gourcy, (2013), los que fueron seguidos por Madrigal, Fonseca; Calderón, Gómez \& Núñez, (2019), aludiendo la necesaria implementación de acciones para la vigilancia de la calidad del agua de los acuíferos como una estrategia a seguir para lograr la sostenibilidad en este sentido.

Cassio Madrazo \& Sánchez Ortiz (2018, p. 186), precisan que las cuencas hidrológicas o hidrográficas son áreas consideradas unidades territoriales básicas para la planeación, gestión y manejo sustentable de los recursos naturales. En este orden Sánchez, Carriel, \& Castillo Ortega, (2021), han determinado la importancia que tiene precisar el alcance de esa gestión, indicando que realmente donde podemos apreciar con mayor exactitud los variados usos de la tierra y las bondades que genera es precisamente en las microcuencas, 
convirtiéndose estos en el sitio más conveniente y adecuado para el ordenamiento y e intervención de la misma.

Por tanto en este contexto, no solo basta con delimitar físicamente las áreas a intervenir, sino también gran importancia reviste gestionar adecuadamente los factores que interceden en la cuenca, tales como la manera de utilización de los recursos hídricos en los procesos de industrialización, producción de alimentos y bebidas, generación de energía, actividades turísticas, minería, agricultura, entre otras, sin dejar de citar el crecimiento de la población así como el impacto que genera el cambio climático.

Al decir de Basualto, Del Valle, Gil, Figueroa, (2018), significa que todas las actividades o acontecimientos que de cualquier forma impacten en la cuenca podrán influenciar o repercutir aguas abajo.

Específicamente respecto al efecto del cambio climático sobre los recursos hídricos de las cuencas hidrográfica, el Informe del Banco Mundial (2014), señala que planificar a largo plazo los recursos hídricos y realizar una coherente gestión de las cuencas, trae como resultados positivos la obtención de beneficios para el abastecimiento de agua, la agricultura, la acuicultura y los ecosistemas, y adicionalmente ayuda a reducir la intensidad de las inundaciones o la exposición a sequías.

Es por ello que cuando nos referimos a esa adecuada gestión de los factores que inciden en la cuenca, como explica Pérez-Ortega, et al., (2016), un aspecto trascedente lo constituye el establecer a través de índices e indicadores, el efecto que generan los cambios en el uso de la tierra y las actividades antrópicas sobre el medio ambiente incluyendo a los recursos hídricos.

Refiriéndose a ello, Saavedra (2018), indica que estos factores necesariamente tienen que gestionarse en el contexto y enfoque de cuenca, debiéndose orientar en acciones integrales de coordinación y consenso entre aquellos actores que localmente conforman la microcuenca.

Gran relevancia tiene en este aspecto el rol que juegan los actores que conforman la microcuenca, integrados como participación ciudadana, mediante la oferta de oportunidades a las comunidades para que se incorporen al programa de vigilancia y a los procesos de gestión de los recursos hídricos. Un ejemplo de ello, es citado por (MadrigalSolís et al., (2019), al referir que la red de monitoreo, es utilizada como una herramienta de gestión participación, que es usada para establecer estrategias y políticas para proteger y conservar el recurso hídrico, lo cual mejora la gestión y el manejo de las aguas, lo cual permite previamente el control de los procesos de contaminación.

Al mismo tiempo, se concuerda con los autores precedentes, al explicar que la gestión participativa, ofrece un campo para la concientización a los habitantes y usuarios de la 
cuenca, respecto a la importancia de las acciones que deben realizar, para que de esa forma contribuyan con un manejo racional y sostenible del recurso hídrico de la zona.

Basado en lo anterior y considerando la importancia que tiene de manera general el manejo adecuado de los recursos ambientales que conforman las microcuencas como factor determinante para el desarrollo integral de las misma; En el caso de Ecuador dada las propias características geográficas y ambientales lo citado adquiere una importancia transcendental, máxime si se tiene en cuenta que un adecuado uso de los recursos naturales y ambientales además de sr necesario para la preservación de la vida en la tierra contribuye al mejoramiento de la calidad de vida de todas las especies y a la sostenibilidad de los ecosistemas. Siguiendo estas ideas cabe preguntarse ¿Cómo potenciar desde bases científicas la utilización adecuada de los recursos ambientales y a su vez favorecer el desarrollo integral de la microcuenca objeto de estudio?

En atención a lo señalado en la presente investigación se parte de considerar las características actuales que presenta la microcuenca del Río Blanco Parroquia Quimiag, cantón Penipe, Provincia de Chimborazo, en Ecuador, lo cual devela la necesidad de buscar alternativas que garanticen en el menor tiempo posible el uso sustentable de la microcuenca de referencia; de ahí que el objetivo del trabajo se centró en aportar un plan de manejo ambiental, que permita de manera paralela la protección de los recursos hídricos de la microcuenca y a su vez tribute al desarrollo local sostenible.

\section{Metodología.}

\subsection{Enfoque, tipo, métodos e instrumentos.}

Para el proceso de investigación se siguió una metodología con enfoque cualitativo de tipo descriptiva no experimental, con base en el estudio bibliográfico y campo. Dentro de los métodos empleados se encuentran métodos teóricos tales como el histórico -lógico, el analítico -sintético, inductivo-deductivo y el sistémico-estructural funcional. Como parte de los métodos empíricos utilizados ocupó un lugar fundamental la observación, mediante la cual se pudo constatar la presencia del problema de investigación, analizando los antecedentes históricos hasta llegar a plantear la estructura de la propuesta a seguir.

Para el diagnóstico inicial se utilizaron materiales e instrumentos tales como: cartas topográficas, flexómetro, embudos, botellas plásticas, envases estériles, fundas ziploc, barreno, prensa para muestras de vegetación, hojas de registro de datos, marcador permanente, probeta. En virtud de tener mayor objetividad en la interpretación de los diferentes resultados fue necesario utilizar además computadoras, Impresoras, Cámaras fotográficas, GPS, estación meteorológica portátil, Caudalímetro de molinete, cronómetro, termómetro de agua, entre otros instrumentos. 


\subsection{Contextualización de la Investigación.}

La investigación se realizó dentro de la zona alta de la microcuenca del "Río Blanco" Parroquia Quimiag, cantón Penipe, Provincia de Chimborazo, en Ecuador. La zona estudiada, se encuentra a una altitud que va desde los 3107 hasta los $4324 \mathrm{msnm}$, y tiene una superficie de 14494.89 hectáreas. Se pudo observar que en la microcuenca se encuentran zonas altas de páramo, en las cuales se presenta las principales fuentes de agua que son utilizadas para consumo humano y bebedero de animales, de la parroquia. Como el sitio de estudio se encuentra en la zona alta, no existen actividades agrícolas cerca del sector.

Como parte de la caracterización de la zona estudiada es importante mencionar que en el área intervenida de la Microcuenca existen varios tipos de zonas climáticas, debido a la influencia de los vientos originados en la Amazonía y al efecto que produce el gradiente altitudinal. La temperatura de la zona de estudio varía entre $\operatorname{los} 7$ a $13.5^{\circ} \mathrm{C}$, la humedad relativa se encuentra dentro del rango de $65-100 \%$. Generalmente en el páramo el clima predominante es frío de alta montaña.

\subsection{Procedimiento metodológico seguido.}

El proceder investigativo contempló varias etapas

\section{Etapa No 1 Constatación y diagnóstico del problema objeto de investigación.}

En esta etapa se realizó un estudio de Línea Base y un diagnóstico inicial, para determinar el estado en el que se encuentra la Microcuenca. Este estudio fue dirigido a identificar los impactos más significativos con el objetivo de ordenar y regular las actividades antropogénicas relacionadas con el sitio ecológico, a fin de dirigir las mismas en función de mejorar la calidad y cantidad de los recursos hídricos necesarios para su desarrollo, y primordialmente necesario para la sobrevivencia de los ecosistemas y agroecosistemas que interaccionan con la Microcuenca.

\section{Etapa No 2 Determinación de los objetivos y estrategia a seguir}

En la segunda etapa una vez realizado y analizados los resultados del diagnóstico inicial se determinan los objetivos de la investigación y se procede a elaborar la estrategia de intervención a seguir, identificándose las etapas, los componentes y estructura del programa a diseñar.

\section{Etapa No 3. Diseño del Programa de Manejo Ambiental}

La tercera etapa se centró en el diseño del Programa o propuesta, para esto se partió de considerar los sub planes existentes, los cuales resultaron de gran utilidad para la elaboración definitiva del Programa de Manejo Ambiental. 


\section{Resultados}

Como resultado de la investigación realizada se logra la elaboración del Programa de Manejo Ambiental, el cual se destinó a proveer una guía de programas, procedimientos, prácticas y acciones orientados a prevenir, eliminar, minimizar y controlar los impactos potencialmente negativos, que las actividades antrópicas desarrolladas en la Microcuenca ocasionan en el entorno ambiental, así como potencializar aquellos aspectos positivos.

Dicho Programa de Manejo Ambiental, quedó conformado por tres sub planes, los cuales permitieron asumir la integralidad del problema investigado desde una posición científica y profunda acerca de las vías más idóneas para su desarrollo e intervención.

Cada sub plan siguió como estructura la declaración del aspecto ambiental, acciones, indicador de cumplimiento, medios de verificación, responsable de la medida y presupuesto.

\section{Figura No1 Sub planes que integran el Programa de Manejo Ambiental}

- Manejo y conservación de Recursos Naturales

\begin{tabular}{|c|c|}
\hline No 1 & - Manejo y conservación de Recursos Naturales \\
\hline $\begin{array}{l}\text { PLAN } \\
\text { No } 2\end{array}$ & - Capacitación y Concientización \\
\hline PLAN & -Seguimiento Ambiental \\
\hline
\end{tabular}

Elaborado por: Nuñez, MS ( 2016 )

\section{Ejemplo Sub Plan No 1}

\section{Plan $\mathbf{N}^{0} 1$}

\section{Manejo y Conservación de Recursos Naturales}

Aspecto ambiental: Agricultura, Ganadería, Pastoreo, Pesca y Turismo.

Objetivo: Mantener la protección al área de influencia directa de la Microcuenca del Río Blanco, mediante la colaboración y el apoyo de la población beneficiaria, para con la preservación de la calidad del recurso hídrico y garantizar la disponibilidad permanente del mismo.

Meta: Ejecutar actividades para la conservación de los recursos naturales de la Microcuenca del Río Blanco, para mantener la permanencia de los servicios ambientales que ofrece el recurso hídrico, protegiendo de igual forma la existencia de especies de flora y fauna propias del sitio.

\section{Actividades}

- Ejecutar visitas con los pobladores del área circundante de la Microcuenca del Rio Blanco, a fin de evidenciar el estado en el que se encuentra la misma, para poder de esta manera mantener las condiciones actuales de conservación de flora, fauna y recurso hídrico de la cuenca. 
Plan $\mathbf{N}^{\mathbf{0}} 1$

Manejo y Conservación de Recursos Naturales

- Mantener los sembríos de vegetación propia de la zona (bosque nativo) que sirven de barreras vivas en las riberas de los ríos que alimentan a la Microcuenca, las mismas que impiden el paso de animales (ganado bovino) a las fuentes hídricas.

- Establecer senderos fijos mediante señalización, a fin de que los sitios que no comprenden caminos de acceso a la Microcuenca no presenten alteraciones y se mantengan vírgenes al acceso de turistas y pobladores que en tiempo futuro pudieran degradar el ecosistema.

\section{Ejecución}

- Las visitas al área circundante la de Microcuenca se realizarán mensualmente, considerando a los representantes de las comunidades beneficiadas por el recurso Hídricos, es decir 13 representantes de las comunidades de Susutul, Gabiñay, Tarau, Torcasa, Balabug, Candelaria, La Loma, Chañag San Franciso, Chañag San Miguel, Releche, Verde Pamba y Santiago de Sali, quienes serán acompañados por los técnicos del Departamento de Gestión Ambiental del Municipio de Penipe, a fin de mostrar a los representantes el estado conservado en el que se encuentra la Microcuenca y concientizarlos para que sus actividades de desarrollo en un futuro no afecten al recurso hídrico.

- Semestralmente los técnicos ambientales del Municipio deberán monitorear el estado del crecimiento y abundancia del bosque nativo, para poder mantener las barreras vivas que se encuentran en la Microcuenca, y poder llevar un índice de crecimiento de flora a lo largo del tiempo.

- Se colocará señalización en los senderos ya establecidos para el tránsito de animales, pobladores y posibles turistas, a los senderos ya presentes el Municipio estará encargado de dar mantenimiento a fin de evitar la necesidad de crear nuevos caminos de acceso que afecten al área de la Microcuenca, dichos senderos deberán prevenir un tránsito de 20 personas al mes entre pobladores y turistas.

- Todas estas actividades serán ejecutadas dentro del área de la Microcuenca del Río Blanco lo que concierne a trabajo de campo, y en la sala de capacitaciones del Municipio lo relacionado a actividades de concientización en la conservación de Microcuencas.

\section{Materiales a emplearse}

- GPS

- Estación Meteorológica portátil

- Equipo de análisis de agua portátil

- Cámara fotográfica

- Check list para análisis del estado de vegetación (barreras vivas) de la Microcuenca.

\section{Beneficiarios directos:}

- Autoridades del Municipio de Penipe, les proporcionará una mejor gestión de los recursos naturales presentes dentro de su jurisdicción.

- Pobladores de las 13 comunidades que se encuentran dentro del área por donde fluye la Microcuenca del Río Blanco.

\section{Beneficiarios Indirectos}

- Poblaciones que se encuentran alejados del cantón Penipe, pero que utilizan el agua proveniente de la Microcuenca del Río Blanco que alimenta a la Cuenca del Pastaza.

\section{Medio de verificación}

- Medición in situ, reconocimiento fotográfico.

- Hojas técnicas de recopilación de datos metereológicos.

- Datos estadísticos de cobertura vegetal de barreras vivas.

Indicadores

- Nombre del Indicador 


\begin{tabular}{|c|c|}
\hline Manejo y Conservacio & Naturales \\
\hline $\begin{array}{l}\text { Conservación de la Microcuenca } \\
\text { - } \quad \text { Descripción } \\
\text { Relación entre la cantidad de bosque } \\
\text { cabo de un año } \\
\text { - } \quad \begin{array}{l}\text { Objetivo del Indicador } \\
\text { Controlar la cobertura vegetal de la }\end{array} \\
\text { - Fórmula de Cálculo } \\
\quad \text { Especies de Bosque Na } \\
\text { Especies de Bosque Nati } \\
\text { - Unidad de Medición } \\
\text { Porcentaje } \\
\text { Meta Prevista } \\
70 \%\end{array}$ & $\begin{array}{l}\text { e al momento y la cantidad al } \\
\text { Lente } \\
\text { un año Blanco } \\
\text { Lño }\end{array}$ \\
\hline $\begin{array}{l}\text { Responsables Directos: Departamento de Ge } \\
\text { Responsables Indirectos: Representantes Co }\end{array}$ & $\begin{array}{l}\text { al del Municipio de Penipe. } \\
\text { zona de estudio. }\end{array}$ \\
\hline Costo estimado de la medida: & \\
\hline Actividad & Costo (\$) \\
\hline $\begin{array}{l}\text { Utilización del transporte Municipal para } \\
\text { recorridos por la zona }\end{array}$ & 20 (Combustible) \\
\hline $\begin{array}{l}\text { Adquisición de una estación meteorológica } \\
\text { portátil }\end{array}$ & 250 \\
\hline Actividad & Costo $(\$)$ \\
\hline $\begin{array}{l}\text { Mantenimiento y calibración del equipo } \\
\text { portátil de medición de parámetros de agua }\end{array}$ & 200 \\
\hline $\begin{array}{l}\text { Mantenimiento adecuado de las barreras } \\
\text { vivas de bosque nativo (limpieza del área) }\end{array}$ & 50 \\
\hline Total & 520 \\
\hline
\end{tabular}

Elaborado por: Nuñez, MS (2016)

De igual manera y como parte de la planificación se determinó previamente el cronograma a seguir para la implementación del Programa de Manejo Ambiental, definiéndose el mismo para 6 cuatrimestres.

Tabla No 1 Cronograma de aplicación del Programa de Manejo Ambiental

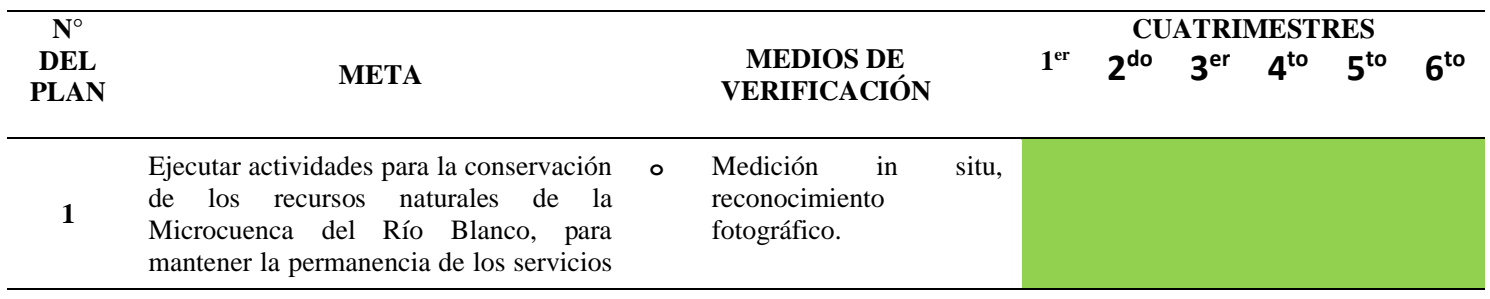


ambientales que ofrece el recurso hídrico, protegiendo de igual forma la existencia de especies de flora y fauna propias del sitio.

Ayudar al uso racional de los recursos naturales presentes en la Microcuenca con la aplicación de un programa de Educación Ambiental a las Autoridades Municipales y pobladores de la zona, para crear una conciencia ambiental que permite mantener una armonía entre la convivencia de la población y el ambiente.
Organización y ejecución de las medidas propuestas en el Plan de Manejo Ambiental, a fin de promover la conservación y el uso sustentable de recurso hídrico, y fomentar la base para futuras investigaciones.

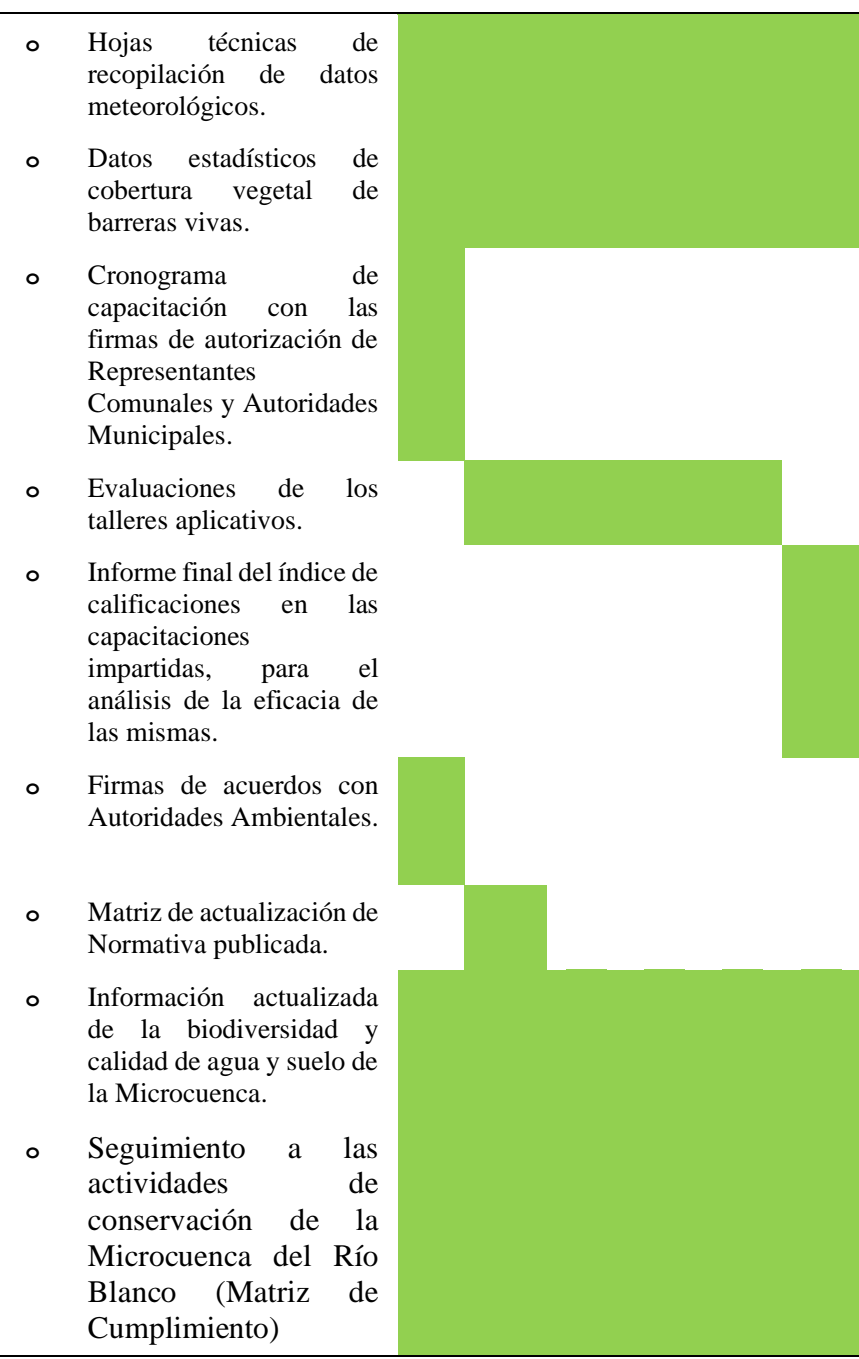

Elaborado por: Nuñez, MS ( 2016 )

Como puede apreciarse el desarrollo de la investigación desarrollada concretamente asume como principales resultados los siguientes:

1. Se corrobora la existencia del problema objeto de estudio en relación con la necesidad de buscar alternativas que garanticen en el menor tiempo posible el uso sustentable de la microcuenca de referencia.

2. Se aporta un Programa de Manejo Ambiental, que dada su estructura, conformación y cronograma previsto para su implementación permite de manera paralela la protección de los recursos hídricos de la microcuenca "Río Blanco" y a su vez tributa al desarrollo local sostenible cumpliéndose de este modo con una de las acciones más prioritarias en la actualidad.

\section{Conclusiones}

- Se concluye que la conservación y manejo adecuado de los recursos ambientales además de ser en extremo prioritario requiere de un proceso de intervención oportuno, el cual debe estar sustentado en un Plan de Manejo Ambiental, de 
carácter integral, que favorezca no sólo la protección de los recursos hídricos, sino también la sostenibilidad y desarrollo local de la microcuenca Río Blanco”, ello permitirá además mejorar la conservación del estado y disponibilidad de los recursos naturales y la supervivencia de las diferentes especies que conforman ese ecosistema incluyendo la vida humana.

\section{Referencias Bibliográficas}

Arteta, Y., Moreno, M Steffanell, I., (2015), La Gestión Ambiental de la Cuenca del Río Magdalena desde un Enfoque Socialmente Responsable Revista Amauta Universidad del Atlántico Barranquilla (Col.) No. 26• Jul-Dic 2015•193-218

Banco Mundial, (2014), Informe sobre Gestión de los recursos hídricos: Resultados del sector. https://www.bancomundial.org/.

Baran, N. y Gourcy, L. (2013). Sorption and minera-lization of S-metolachlor and its ionic meta- bolites in soils and vadose zone solids: Conse-quences on groundwater quality in an alluvialaquifer (Ain Plain, France). Journal of Contaminant Hydrology, 154: 20-28. Doi: https://doi.org/10.1016/j.jconhyd.2013.07.009.

Basualto S, Del Valle J, Gil M, Figueroa R, (2018)- Modelos de gestión, conflictos y mediación en cuencas hidrográficas: los casos de España y Brasil y su aplicabilidad a Chile. Aqua-LAC Volumen 11 (1) Septiembre 2018 - Marzo 2019 doi: 10.29104/phi-aqualac/2019-v11-1-05 ISSN 1688-2873.

Cassio Madrazo, E., \& Sánchez Ortiz, E. (2018). Gobernanza ambiental para el desarrollo sostenible de la cuenca de Santiaguillo, Durango Environmental governance for sustainable development of Santiaguillo's basin , Durango. Espiral, xxv (72), 183208.

López, W., (2014), Análisis del manejo de cuencas como herramienta para el aprovechamiento sustentable de recursos naturales. Revista Chapingo Serie Zonas Áridas, vol. XIII, núm. 2, 2014, pp. 39-45 Universidad Autónoma Chapingo Durango, México Disponible en: http://www.redalyc.org/articulo.oa?id=455545055001

Madrigal-Solís, H.; Fonseca-Sánchez, A.; Calderón-Sánchez, H.; Gómez-Cruz, A. \& Núñez-Solís, C. (2019). Design of a monitoring network as a participative management tool: physical and chemical quality of groundwater in three subbasins in the Central Valley of Costa Rica. Revista Uniciencia. 33(1), 43-60. Doi: http://dx.doi.org/10.15359/ru.33-1.4

Nuñez, MS., (2016) Propuesta del Plan de Manejo Ambiental para el uso sustentable de la Microcuenca del cantón Penipe, Chimborazo, Ecuador. Tesis en opción al grado Magister en Ciencias Ambientales. Facultad de Ciencias Naturales y Matemáticas 
Departamento de Ciencias Químicas Y AMBIENTALES Escuela Superior Politécnica del Litoral. ESPOL. Guayaquil.

Pérez-Ortega Dj, Pérez D, Américo J, Carvalho S, Segovia J (2016). Development of Index of Resilience for Surface Water in Watersheds. Journal of Urban and Environmental Engineering, v.10, n.1, p.72-82.

Sánchez Rodríguez, A. N., Carriel Bustamante, V. V., \& Castillo Ortega, Y. (2021). Modelo de gestión sostenible de los recursos hídricos de la microcuenca alta del río Santa Rosa. Ciencia Digital, 5(1), 182-196. https://doi.org/10.33262/cienciadigital.v5i1.1532

Saavedra, C. 2018. Cuencas sostenibles: Fundamentos y recomendaciones. Proyecto gestión integral del agua de la Coperación suiza en Bolivia. HELVETAS Swiss Intercooperation. 44 pág.

Sepúlveda, S. (2002). Desarrollo Sostenible Microregional. Métodos para Planificación Local. IICA-UNA. San José, Costa Rica. 313 p.

Van den Brink, C.; Frapporti, G.; Grif $\square$ oen, J. y Jan Zaadnoordijk, W. (2007). Statistical analysisof anthropogenic versus geochemical-contro-lled differences in groundwater compositionin The Netherlands. Journal of Hydrolog, 336, 470-480. Recuperado de https://doi.or-g/10.1016/j.jhydrol.2007.01.024 


\section{PARA CITAR EL ARTÍCULO INDEXADO.}

Nuñez Moreno, M. S., \& Calderón Tapia, C. G. (2021). Uso sustentable de la Microcuenca del Río Blanco: Posibles soluciones desde un Programa de Manejo Ambiental.

\section{\Ciencia}

El artículo que se publica es de exclusiva responsabilidad de los autores y no necesariamente reflejan el pensamiento de la Revista Conciencia Digital.

El artículo queda en propiedad de la revista y, por tanto, su publicación parcial y/o total en otro medio tiene que ser autorizado por el director de la Revista Conciencia Digital.

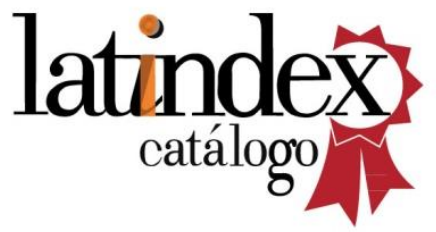

